\title{
Qualidade de vida de crianças e adolescentes: uma revisão bibliográfica
}

\author{
Quality of life of children and adolescents: a bibliographical review
}

\author{
Ana Helena Rotta Soares ${ }^{1}$ \\ Antilia Januária Martins ${ }^{1}$ \\ Maria da Conceição Borges Lopes ${ }^{1}$ \\ José Augusto Alves de Britto ${ }^{1}$ \\ Cristiano Queiroz de Oliveira ${ }^{1}$ \\ Martha Cristina Nunes Moreira ${ }^{1}$
}

${ }^{1}$ Departamento de

Ensino, Instituto Fernandes

Figueira, Fundação Oswaldo

Cruz. Av. Rui Barbosa 716,

Flamengo. 22450-000

Rio de Janeiro RJ.

ahrsoares@iff.fiocruz.br

\begin{abstract}
The scope of this article was to analyze Brazilian scientific articles that assess the quality of life related to the health of children and adolescents. The databases consulted were SciELO and Bireme involving a total of 30 articles published between 1990 and 2008. The articles confirmed the predominance of quantitative methodologies (70\%) with a high incidence of applications of HRQL (66.7\%) and generic texts (28.6\%). The concept of quality of life was evaluated from two standpoints: in the context of scientific research and as part of the desired results of healthcare and public policy. This construct is presented from a multidimensional perspective, embracing the social, psychological, physical and individual dimensions, which is based on the premise that the individuals themselves must assess their own quality of life. The article draws attention to the need to create and use instruments of quality of life for children and adolescents that enhance their perspective about their health processes using appropriate tools for each phase of development. The assessment of quality of life should be incorporated in clinical assessment since chronic illness has repercussions in the many dimensions of the life.
\end{abstract} Key words Quality of life, Child health, Adolescent health
Resumo O presente artigo tem como objetivo analisar as produções científicas nacionais, que abordam a temática da qualidade de vida ligada à saúde da criança e do adolescente. As bases de dados consultadas foram a SciELO e Bireme totalizando 30 artigos publicados no período de 1990 a 2008. Revelou-se uma predominância da abordagem quantitativa (70\%) e um alto indice de aplicações de instrumentos de avaliações de qualidade de vida ligada à saúde (66,7\%) e genéricos $(28,6 \%)$. O conceito de qualidade de vida foi usado em duas vertentes: no contexto da pesquisa científica e como parte dos resultados desejados das práticas assistenciais e políticas públicas. O referido construto é apresentado através de uma perspectiva multidimensional, incluindo no mínimo as dimensões fisica, psicológica e social e de uma perspectiva subjetiva, o que implica no pressuposto de que a própria pessoa deve avaliar sua qualidade de vida. Observou-se ainda um movimento de valorização da perspectiva da criança e do adolescente como relator de sua experiência de vida. Conclui-se pela necessidade de criação e utilização de instrumentos de avaliação de qualidade de vida que valorizem a perspectiva de crianças e adolescentes e adequados a sua fase de desenvolvimento. A avaliação de qualidade de vida deve ser incorporada à avaliação clínica uma vez que a doença crônica repercute nas diversas dimensões da vida dos mesmos.

Palavras-chave Qualidade de vida, Saúde da criança, Saúde do adolescente 


\section{Introdução}

A década de 90 foi marcada por debates acadêmicos e políticos sobre a definição do conceito de qualidade de vida, principalmente referidos a identificação das dimensões contempladas para o mesmo e a preocupação quanto ao desenvolvimento de instrumentos de avaliação que considerem a perspectiva do sujeito. As primeiras publicações nacionais sobre qualidade de vida aconteceram em 1993 e se concentram em aspectos relacionados à volta ao trabalho após intervenções cirúrgicas e à falta de oportunidades profissionais devido à doença crônica e nutrição infantil ${ }^{1,2}$.

Consoante com o interesse mundial pelo conceito, a comunidade científica brasileira aquece o debate sobre os significados e possíveis métodos de avaliação de qualidade de vida através do crescente desenvolvimento de produções científicas em diferentes áreas do saber como: medicina, educação, psicologia, enfermagem e economia. A ampliação do interesse da comunidade científica brasileira está refletida na aceleração de produções ligadas ao tema. Segundo Soares ${ }^{3}$, em novembro de 2004 a SciELO citava 81 produções científicas nacionais relacionadas à qualidade de vida. Já em outubro de 2007 a mesma base de dados demonstrava 274 produções.

A despeito do grande crescimento de produções que contemplam o conceito de qualidade de vida, aquelas ligadas à saúde da criança e do adolescente, no seu sentido ampliado, no Brasil ainda são escassas. Avaliar a qualidade de vida de crianças sem nenhum acometimento de saúde e aquelas com doenças crônicas torna-se cada vez mais importante, já que o incremento nas tecnologias médicas contribui para o aumento da sobrevida dessa última parcela, o que pode não significar a promoção da qualidade de suas vidas.

Este estudo pretende apresentar uma revisão bibliográfica de produções científicas nacionais que abordam a temática da qualidade de vida ligada à saúde da criança e do adolescente, analisando os conteúdos gerados pela comunidade científica brasileira. A revisão objetiva rastrear as produções da saúde da criança e do adolescente que empregam o conceito de qualidade de vida tanto em sua aplicação genérica, isto é, ligada à posição do individuo no mundo e sua experiência de vida, quanto no sentido específico relacionado às doenças ou intervenções em saúde.

\section{Metodologia}

O presente trabalho baseou-se no referencial da pesquisa bibliográfica, que consiste no exame da literatura científica para levantamento e análise do que já se produziu sobre determinado tema. Envolveu as atividades básicas de identificação, compilação, fichamento, análise e interpretação ${ }^{4}$. O período da pesquisa foi de 1990 a 2008. O recorte temporal se dá em função dos debates acadêmicos e políticos sobre a definição do conceito de qualidade de vida na década de 90 na intenção de ampliar o conceito de saúde através de uma perspectiva subjetiva e composta por dimensões tanto positivas quanto negativas do processo de adoecimento.

Primeiramente foi feita uma revisão bibliográfica das produções científicas em saúde no Brasil, através de um levantamento na biblioteca eletrônica SciELO, que abarca uma compilação selecionada de periódicos científicos brasileiros. Foram encontradas 370 referências através da palavra-chave "qualidade de vida". Contudo, ao se acrescentar as palavras-chave "infância" e "adolescência" este número foi reduzido para quatro. Visando ampliar a qualidade da busca e identificar o número real das publicações que tinham como população em questão crianças e adolescentes, os resumos dos 370 artigos foram rastreados em busca de produções que discutiam exclusivamente a qualidade de vida de crianças e adolescentes. Foram localizados 30 artigos.

Com o propósito de ampliar os canais de divulgação foi efetuada uma revisão das publicações na área de saúde, através da Biblioteca Virtual Bireme, tendo sido consultadas as bases de dados Lilacs e Adolec utilizando as mesmas palavras-chave. Foram encontradas 5089 referências na Lilacs através da palavra-chave "qualidade de vida" e 1432 referências na Adolec por meio da mesma palavra-chave. Na inclusão das palavras "infância" e "adolescência" estes números caíram para 23 e 19 respectivamente.

A organização das produções demonstrou algumas sobreposições levando ao número final de 33 , sendo 3 deles publicados na língua inglesa ${ }^{5-7}$, e 2 publicados na língua espanhola ${ }^{8,9}$. Destes artigos, foram excluídos aqueles que abordavam a qualidade de vida de populações não brasileiras ${ }^{8-}$ ${ }^{10}$. A exclusão dos artigos que tratavam do tema a partir de experiências em outros países se deu em função da necessidade de construir uma aproximação exploratória com o estado da arte das pesquisas no Brasil. Considerando para tanto, no interior da análise empreendida, a maneira como 
os autores conceituam qualidade de vida, a utilização ou não de instrumentos específicos para determinadas patologias referidas a segmentos da população infanto-juvenil, ou ainda instrumentos genéricos ligados à saúde da população de crianças e jovens sem nenhuma patologia identificada. No território brasileiro interessou-nos também observar o processo de utilização de instrumentos validados ou não para a língua portuguesa. Foram incluídos apenas os artigos originais em função da sua maior circulação no meio acadêmico e profissional, excluindo assim, teses de doutorado, dissertações de mestrado, monografias, anais de congresso, editoriais e artigos que não continham "qualidade de vida" como descritor, o que totalizaram 18 produções.

A análise dos dados foi realizada seguindo as perspectivas da técnica de Análise de Conteúdo, na modalidade Temática ${ }^{11}$. Inicialmente foi feita uma leitura flutuante das produções, para em seguida serem identificados os eixos temáticos e estabelecidos os núcleos de sentido.

\section{Resultados e discussão}

\section{Caracterização do acervo revisado}

Durante a revisão das publicações alguns aspectos foram identificados como relevantes no processo de análise dos conteúdos. Desta maneira os artigos foram agrupados refletindo o objetivo geral de cada publicação nos seguintes eixos temáticos: (1) avaliação da qualidade de vida, (2) qualidade de vida como característica de distinção, (3) elaboração de instrumentos e (4) adaptação de instrumentos.

O primeiro eixo temático denominado avaliação da qualidade de vida refere-se aos estudos que possuem objetivo de descrever e analisar a qualidade de vida relacionada à saúde da criança e do adolescente. Vale ressaltar, que o tema engloba as produções que empregam o conceito de qualidade de vida de forma genérica e aquelas que buscam averiguar o impacto de determinadas doenças, agravos e tratamentos na vida de indivíduos.

O segundo eixo temático denominado qualidade de vida como característica de distinção diz respeito aos estudos que não definiram o termo qualidade de vida, mas que o utilizaram de forma implícita, como um objetivo a ser alcançado ou ainda como um selo de boas práticas em saúde.

O terceiro eixo temático nomeado elaboração de instrumentos inclui produções que demonstram os processos de construção de instrumentos para avaliação da qualidade de vida de crianças e/ou adolescentes. É importante frisar que nesta categoria encontram-se tanto instrumentos que respeitaram os processos de verificação da fidedignidade e validade dos instrumentos, como outros elaborados a partir de dados identificados na prática clínica e temas identificados na literatura.

A última categoria identificada como adaptação de instrumentos, refere-se às produções que objetivam a tradução e adaptação para a língua portuguesa e para a cultura brasileira de instrumentos internacionais. Da mesma forma que na categoria anterior, algumas produções incluem o processo de validação e outras não.

O Quadro 1 demonstra a caracterização do acervo estudado, segundo o autor e ano da publicação, distribuído em cada eixo temático.

Quadro 1. Caracterização do acervo estudado nos eixos temáticos, segundo autor e ano da publicação.

\begin{tabular}{|c|c|}
\hline Tema & Autor/Ano \\
\hline $\begin{array}{l}\text { Avaliação da } \\
\text { qualidade de vida }\end{array}$ & $\begin{array}{l}\text { 1) Silva e Leite, } 2006 \\
\text { 2) Maia Filho et al., } 2006 \\
\text { 3) Yacubian-Fernandes et al., } 2007 \\
\text { 4) Anders e Lima, } 2004 \\
\text { 5) Di Francesco et al., } 2004 \\
\text { 6) Faleiros e Machado, } 2006 \\
\text { 7) Barreire et al., } 2003 \\
\text { 8) Feitosa et al., } 2005 \\
\text { 9) Elias e Assumpção Jr, } 2006 \\
\text { 10) Guimarães et al., } 2003 \\
\text { 11) Fernandes e Souza, } 1999 \\
\text { 12) Costa et al., } 2007 \\
\text { 13) Martins et al., } 1996 \\
\text { 14) Cartana, 2005 } \\
\text { 15) Radünz e Olson, } 2005 \\
\text { 16) Brasil et al., } 2003 \\
\text { 17) Favarato e Romano, } 1994 \\
\text { 18) Bampi et al., } 2008 \\
\text { 19) Soares et al., } 2008\end{array}$ \\
\hline $\begin{array}{l}\text { Qualidade de vida } \\
\text { como característica } \\
\text { de distinção }\end{array}$ & $\begin{array}{l}\text { 1) Costa e Bigras, } 2007 \\
\text { 2) Monteiro et al., } 1993 \\
\text { 3) Chaves et al., } 2007 \\
\text { 4) Dóro et al., } 2003 \\
\text { 5) Oria, } 1988\end{array}$ \\
\hline $\begin{array}{l}\text { Elaboração de } \\
\text { instrumentos }\end{array}$ & $\begin{array}{l}\text { 1) Maia Filho et al., } 2005 \\
\text { 2) Souza, } 1999\end{array}$ \\
\hline $\begin{array}{l}\text { Adaptação de } \\
\text { instrumentos }\end{array}$ & $\begin{array}{l}\text { 1) Assumpção Jr. et al., } 2000 \\
\text { 2) La Scala et al., } 2005 \\
\text { 3) Novato et al., } 2008 \\
\text { 4) Rozov et al., } 2006\end{array}$ \\
\hline
\end{tabular}


Os artigos também foram classificados segundo a abordagem metodológica utilizada. Para isso, foram identificadas as seguintes categorias: (1) Abordagem quantitativa; (2) Abordagem qualitativa; (3) Revisão bibliográfica; e (4) Ensaio teórico. O Gráfico 1 permite a visualização de tal organização:

$\mathrm{Na}$ revisão dos 30 artigos avaliados revelouse uma predominância da abordagem quantitativa com 21 produções, cerca de 70\%: Silva e Leite $^{12}$; Yacubian-Fernandes et al. ${ }^{13}$; Assumpção Jr. et al. ${ }^{14}$; Elias e Assumpção Jr. ${ }^{15}$; Dóro et al. ${ }^{5}$; Di Francesco et al. ${ }^{16}$; Faleiros e Machado ${ }^{17}$ Rozov et al. ${ }^{18}$; La Scala et al. ${ }^{19}$; Barreire et al. ${ }^{20}$; Feitosa et al. ${ }^{6}$; Maia Filho et al. ${ }^{21}$; Guimarães et al. ${ }^{22}$; Fernandes e Souza ${ }^{23}$; Souza ${ }^{24}$; Costa et al. ${ }^{25}$; Martins et al. ${ }^{26}$; Brasil et al. ${ }^{27}$; Favarato e Romano ${ }^{28}$; Bampi et al. ${ }^{29}$; Novato et al. ${ }^{30}$. Somente 4 trabalhos, cerca de $13,3 \%$, abordam a qualidade de vida através da metodologia qualitativa: Anders e Lima ${ }^{31}$; Cartana $^{8}$; Radünz e Olson ${ }^{32}$; Soares et al. ${ }^{33}$. Estes achados são referendados pela literatura especializada e apontam para a tendência de estudos quantitativos e qualitativos sobre qualidade de vida com a predominância dos primeiros ${ }^{34}$. Os demais estudos foram classificados como ensaio teórico e revisão bibliográfica ${ }^{7,35-38}$.

Nos estudos que se propuseram a fazer uma avaliação da qualidade de vida, adaptação de instrumentos e elaboração de instrumentos, revelou-se um alto índice de instrumentos de avaliação de qualidade de vida ligada à saúde $(66,7 \%)$ e genéricos $(28,6 \%)$. Somente um estudo $(4,7 \%)$ utilizou os dois tipos de instrumentos ${ }^{6}$. Tal dado revela uma lacuna no que diz respeito a instrumentos que tornam possível a exploração da qua-

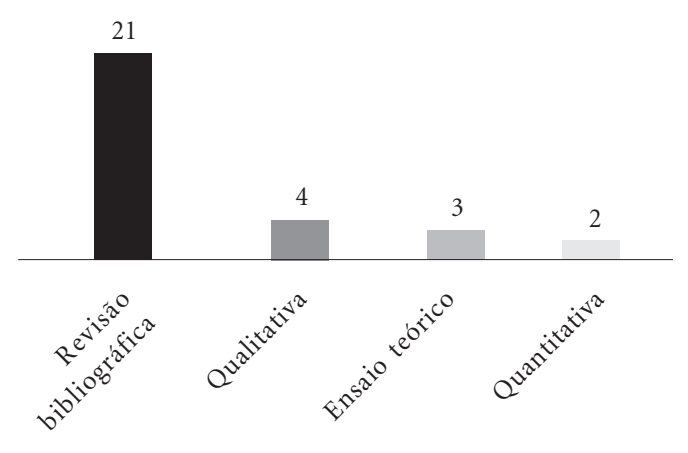

Gráfico 1. Distribuição das produções segundo abordagem metodológica. lidade de vida de crianças de forma ampla e não apenas daquelas em processo de adoecimento.

Para exemplificar os instrumentos de avaliação de qualidade de vida ligada à saúde tem-se o Obstructive Sleep Apnea-18-Item Questionnaire (OSA-18), que é um questionário para avaliação da qualidade de vida de crianças com distúrbios obstrutivos do sono ${ }^{12}$, o Inventário Simplificado de Qualidade de Vida na Epilepsia Infantil ${ }^{23,24}$, os questionários de qualidade de vida em Fibrose Cística $^{18}$, o Pediatric Asthma Quality of Life Questionnaire (PAQLQ-A) ${ }^{19}$, dentre outros.

Quanto aos instrumentos genéricos, o mais utilizado foi o Autoquestionnaire Qualité de Vie Enfant Imagé (AUQEI) ${ }^{6,14,15,20}$. O World Health Organization Quality of Life WHOQOL-Bref ${ }^{29}$, o Child Health Questionnaire-Parent Form 50 (CHQ-PF50) ${ }^{17}$, Parental-Caregiver Perceptions Questionnaire (P-CPQ) ${ }^{6}$, e o Medical Outcomes Study SF-36 Health Survey ${ }^{25}$ são os outros instrumentos de avaliação de qualidade de vida genéricos que foram utilizados.

No que se refere ao processo de desenvolvimento de instrumentos nacionais revelou-se duas produções $^{21,24}$ e à validação de instrumentos internacionais para a língua portuguesa, quatro estu$\operatorname{dos}^{14,18,19,30}$. Ressalta-se que apesar dos esforços acima citados a literatura não demonstrou publicações ligadas a incorporação destas medidas em pesquisas subsequentes ou na prática clínica.

Observa-se que o interesse para a utilização e a criação de instrumentos de qualidade de vida no campo da saúde da criança e do adolescente vem se desenvolvendo de forma gradativa na comunidade científica brasileira. A motivação parte das discussões sobre a importância da inclusão de instrumentos que contemplem a avaliação da qualidade de vida nos serviços assistenciais de forma rotineira. Apesar disso, a escassez de instrumentos nacionais parece refletir a dificuldade da comunidade científica em desenvolver instrumentos de avaliação de qualidade de vida que se apliquem a diversidade sociocultural do país.

A revisão demonstrou que sendo qualidade de vida um conceito dinâmico e multidimensional seu estudo necessariamente requer abordagens provenientes de olhares variados. Desta forma, observou-se uma grande diversidade quanto às categorias profissionais dos autores principais que publicam sobre o tema. É importante ressaltar que em relação às categorias profissionais da área de saúde que publicam sobre a temática da qualidade de vida ligada à infância e adolescência, os artigos demonstram que a Medicina é, indiscutivelmente, a área que reúne mais 
produções, totalizando 12 artigos. Este dado encontra-se consoante com os achados de Oliveira et al. ${ }^{39}$. A Medicina é seguida pela Enfermagem que totalizou 9 publicações e logo a Psicologia com 8 produções. Ainda, foi encontrada uma publicação da Sociologia, uma da Educação e uma da Odontologia. É importante ressaltar que apesar destes números refletirem apenas os dados referidos ao autor principal de cada publicação, as associações entre áreas profissionais observadas dentre as publicações estudadas apontam para uma tendência de equipes multidisciplinares na abordagem da qualidade de vida ligada à infância e adolescência.

\section{Qualidade de vida -}

um conceito multidimensional e subjetivo

Um dos núcleos de sentido identificado foi “a qualidade de vida como um conceito multidimensional e subjetivo". O termo qualidade de vida compreende diversos significados, que refletem os conhecimentos, as experiências e os valores de determinados indivíduos e sociedades, em diferentes épocas e locais, refletindo histórias de vida diferentes ${ }^{11}$. Duas tendências predominam na área da saúde quanto à conceituação de qualidade de vida: "qualidade de vida como um conceito mais genérico, e qualidade de vida relacionada à saúde (health-related quality of life)" 34 .

Na primeira tendência, qualidade de vida tem um sentido mais amplo, mais genérico. Um exemplo que ilustra essa tendência é a concepção da Organização Mundial de Saúde (OMS), que define qualidade de vida como a percepção que o indivíduo tem de sua posição na vida considerando o contexto da cultura e sistemas de valores nos quais ele está inserido, e em relação a seus objetivos, suas expectativas, padrões e preocupações ${ }^{40}$. Este é um conceito amplo que reflete a complexidade do construto e busca inter-relacionar o meio ambiente com os aspectos físicos, psicológicos, nível de independência, relações sociais e crenças pessoais ${ }^{41}$.

O termo qualidade de vida relacionada à saúde tem um sentido mais restrito, que implica os aspectos mais diretamente associados às doenças ou intervenções em saúde. Auquier et al ${ }^{42}$ utilizam o termo qualidade de vida ligado à saúde, que significa o valor que é atribuído à vida, ponderado pelas deteriorações funcionais; as percepções e condições sociais que são induzidas pela doença, agravos, tratamentos e a organização política e econômica do sistema assistencial. Portanto, os estudos referem-se a situações relacio- nadas à qualidade de vida das pessoas após a experiência de doenças ou intervenções médicas, sendo muito comuns nos casos de doenças crônicas como câncer, diabetes, HIV/Aids, transtornos mentais, dentre outras.

As duas tendências conceituais sobre o termo qualidade de vida na área da saúde irão gerar instrumentos específicos: qualidade de vida como um conceito genérico utiliza questionários de base populacional sem especificar patologias e que são "mais apropriados para estudos epidemiológicos, planejamento e avaliação do sistema de saúde" ${ }^{43}$. Além do World Health Organization Quality of Life (WHOQOL), criado pela OMS, há outros instrumentos genéricos de avaliação da qualidade de vida que são muito utilizados nas pesquisas e na clínica: o Medical Outcomes Study SF-36 Health Survey e o Sickness Impact Profile $^{34}$. Quanto aos instrumentos de avaliação da qualidade de vida relacionada à saúde, o European Organization for Research and Treatment of Cancer (EORTC QLQ-C30) e o Medical Outcomes Study-HIV Health Survey (MOS-HIV) ilustram esse tipo de instrumento ${ }^{34}$.

Desde o início da década de 90 há um consenso entre os estudiosos sobre a natureza subjetiva e multidimensional do construto de qualidade de vida ${ }^{34,40}$. A ideia de qualidade de vida não se restringe à satisfação de necessidades materiais, mas também está relacionada a valores nãomateriais como inserção social, felicidade, liberdade e bem-estar entre outros. Surge assim a concepção de que a qualidade de vida somente pode ser avaliada pela própria pessoa e não mais por um observador, como era feito anteriormente. A partir daí passou-se a considerar a percepção da pessoa sobre o seu estado de saúde e outros aspectos não-médicos de sua vida.

Qualidade de vida é vista ainda como um construto multidimensional que inclui, no mínimo, as seguintes dimensões: (1) física, que compreende a percepção do indivíduo sobre sua condição física, (2) psicológica, ou seja, a percepção do indivíduo sobre sua condição afetiva e cognitiva e (3) social, que é a percepção do indivíduo sobre os relacionamentos sociais e os papéis sociais adotados na vida. Além disso, qualidade de vida inclui dimensões positivas, como por exemplo, a mobilidade, e negativas, como a dor ${ }^{40,44}$.

A revisão apresenta a construção do conceito de qualidade de vida através de uma perspectiva multidimensional e subjetiva do construto. Porém, a multidimensionalidade pode apresentar um desafio na abordagem do construto através de domínios universais e não simplesmente da 
organização da experiência da vida em escores exclusivos que podem vir a fragmentar tal experiência. Este desafio pode erroneamente transformar-se em críticas direcionadas à priorização da abordagem quantitativa para a apreciação da qualidade de vida. No entanto, aproximar a qualidade de vida através de uma abordagem qualitativa não necessariamente garante uma perspectiva englobante da vida já que os domínios priorizados para compor o construto podem reproduzir uma visão fragmentada e pré-estabelecida da mesma.

O termo qualidade de vida nem sempre é explicitado nos textos e alguns estudos ${ }^{5,25,36-38}$ apesar de utilizá-lo como palavra-chave, não apresentam uma conceituação. Desta forma, esvazia-se o significado do construto para a criação de definições de qualidade de vida que apontam para estratégias de prevenção e promoção da saúde da criança e do adolescente ou para um objetivo assistencial a ser alcançado. Isto é, o temo qualidade de vida aparece como ou um selo de boas práticas em saúde ou como uma definição de metas a serem alcançadas, no entanto, nestas publicações não se faz menção ao conceito.

A primeira publicação em saúde no Brasil que aborda a qualidade de vida ligada à infância ilustra esta forma de uso do termo. O trabalho data de 1993 e objetiva a descrição da trajetória da nutrição infantil no Brasil nas décadas de 70 e 80 através da comparação de dois inquéritos nutricionais $^{36}$. O termo qualidade de vida aparece apenas como descritor do artigo e sua discussão se dá de maneira implícita. Desta forma, o autor lança mão do conceito como descritor do trabalho para indicar os progressos nutricionais decorrentes das condições favoráveis da saúde infantil.

A análise revelou ainda que o conceito de qualidade de vida foi utilizado em duas vertentes: (1) no contexto da pesquisa científica, com o propósito de avaliar a sensação subjetiva de bem-estar de crianças e adolescentes acometidas por enfermidades, detectando e comparando o impacto psicossocial de condiçõos clínicas e de esquemas terapêuticos nas suas vivências e (2) como parte dos resultados desejados das práticas assistenciais e das políticas públicas.

\section{A criança e o adolescente com doença crônica como relatores de sua qualidade de vida}

Um outro núcleo de sentido identificado foi a criança e o adolescente com doença crônica como relatores de sua qualidade de vida. A necessidade de tratamentos diários longos e complexos leva ao comprometimento do bem-estar físico e social de crianças e adolescentes com doença crôni$\mathrm{ca}^{18}$. Adicionalmente, o aumento da sobrevida destes pacientes e a melhora do prognóstico em doenças crônicas têm mudado o foco das avaliações do tratamento. Conforme mencionado anteriormente, a literatura evidencia um crescente interesse não apenas dos parâmetros clínicos da doença, mas da experiência de adoecimento dos sujeitos e as interferências do processo de adoecimento em suas funções diárias.

A revisão apontou para a aceleração no número de artigos publicados sobre qualidade de vida ligada à infância e a adolescência desde o ano de 2006, 13 artigos no total o que indica cerca de $40 \%$ do acervo. Tal elevação destaca um avanço na saúde da criança e do adolescente, já que objetiva um conhecimento mais amplo do processo de saúde e doença dos mesmos prometendo ser essencial para a melhora do tratamento da mesma. É fundamental que profissionais de saúde compreendam e abordem as necessidades, ansiedades, projetos e sonhos desta população no sentido de facilitar sua compreensão e vivência com e apesar da doença ${ }^{3}$.

Apesar da evidente valorização dos elementos contemplados na avaliação da qualidade de vida em crianças e adolescentes observa-se que a mesma tem sido considerada em algumas ocasiões apenas como uma medida clínica complementar para a monetarização individual de pacientes com doenças crônicas. Não obstante, os elementos abordados nestas avaliações têm suscitado o interesse na criação de instrumentos e validação dos mesmos para diversas línguas nesta última década. A exemplo disso estão os questionários para pacientes com fibrose cística e asma, que juntos à escala de avaliação da qualidade de vida, foram validados para a língua portugue$\mathrm{sa}^{14,18,19}$ e apresentaram boa adaptação para os pacientes brasileiros.

Dentre as publicações desta revisão encontram-se diversas produções sobre a qualidade de vida relacionada a diferentes doenças crônicas, transplantes ou tratamentos de longo curso e destaca-se como trabalho pioneiro os estudos da Faculdade de Ciências Médicas da Universidade Estadual de Campinas (Unicamp) sobre qualidade de vida e epilepsia ${ }^{20,45,46}$. Estas investigações sobre a qualidade de vida de crianças e adolescentes portadores de epilepsia englobam desde a criação de um instrumento quantitativo para crianças, até discussões sobre a qualidade 
de vida de pacientes submetidos a cirurgias e o impacto da epilepsia no trabalho.

O conhecimento produzido por este grupo de pesquisa já serviu como ponto de partida para o desenvolvimento de outras importantes contribuições na área do estudo sobre a epilepsia em crianças e adolescentes, culminando na construção de um questionário multidimensional que analisa a qualidade de vida da criança com epilepsia do ponto de vista dos responsáveis permitindo um tratamento mais eficiente e individualiza$\mathrm{do}^{21}$. É importante sinalizar que dentre as conclusões da aplicação preliminar do instrumento criado, os autores frisam a necessidade de instrumentos que englobem a experiência da doença crônica na infância de maneira universal no intuito de conhecer e enfrentar as limitações ao crescimento pessoal e social que a mesma apresenta.

Verificou-se ainda nas publicações, que 14 (50\%) avaliações da qualidade de vida de crianças e adolescentes fazem-se através da perspectiva de seus cuidadores ${ }^{6,8,9,12,13,16,17,21-24,27,32,47}$. Dentre os artigos revisados, 12 avaliações, cerca de $43 \%$, fazem-se através da perspectiva da criança e/ou do adolescente sobre sua qualidade de vida $^{5,10,14,15,19,26,28-31,33,34}$ e 2 avaliações utilizam uma combinação das perspectivas das crianças e adolescentes e seus cuidadores ${ }^{18,20}$.

Com relação à ainda incipiente participação e consideração das crianças e adolescentes como sujeitos de pesquisas no campo da qualidade de vida e saúde, podemos apontar 3 possíveis fatores: 1) a interferência dos desafios éticos à inclusão dessa população; 2) a mudança cultural no processo de diagnóstico e tratamento da saúde de crianças e adolescentes, que os incluam e valorizem suas participações; 3 ) a criatividade do pesquisador na adaptação de instrumentos promotores da participação do segmento infanto-juvenil. Primeiramente, a participação desse segmento infanto-juvenil necessariamente considerado vulnerável ${ }^{48}$ implica a inclusão de seus responsáveis no processo de consentimento e negociação da participação, a fim de proteger seus interesses. Por outro lado, o pesquisador precisa reconhecer que o segmento de jovens e crianças é capaz de desenvolver um autoconhecimento sobre seu processo saúde-doença, e de comunicá-lo.

A inclusão de crianças e adolescentes como sujeitos de pesquisa e na produção de conhecimento, depende, portanto, da capacidade dos mesmos serem desde sempre incluídos, respeitados seus limites e capacidades, no processo complexo de diagnóstico/tratamento e produção de saúde ${ }^{49}$. Além disso, no que concerne ao proces- so específico de fazer uma pesquisa, é necessária a habilidade e criatividade do pesquisador em adaptar instrumentos que se tornem interessantes e mobilizem crianças e adolescentes a socializarem suas experiências ${ }^{49}$.

Muito embora, observe-se um movimento de valorização da perspectiva da criança e do adolescente como relator de sua experiência de vida pela comunidade científica, verificou-se que algumas produções incluíam como possível público para seus instrumentos desde adolescentes até idosos ${ }^{26,29}$ deflagrando a não consideração das especificidades desta fase de desenvolvimento, ignorando que o conceito de qualidade de vida é composto de dimensões configuradas em função da posição do sujeito no mundo, sua experiência cultural, histórica, subjetiva e social.

A revisão provoca uma reflexão acerca da posição das crianças e dos adolescentes e suas necessidades, expectativas e vivências específicas. Fica claro através da análise das produções que o estudo da qualidade de vida na infância e na juventude, seja esta portadora de doenças ou agravos ou submetida a tratamentos de ordem biomédica, requer a aplicação de uma metodologia que esteja atenta às particularidades de cada período da vida, suas experiências, necessidades e transformações. Ou seja, conforme os resultados de algumas publicações, estudar a qualidade de vida de jovens ignorando temas centrais desta etapa da vida como sexualidade, busca de inserção social e grupalidade é impossível ${ }^{3,33}$.

\section{Considerações finais}

Ao final da análise aqui empreendida assinala-se que o surgimento, utilização e apropriação do construto qualidade de vida demarcam um avanço significativo na inclusão do processo saúdedoença na perspectiva ampliada e situacional do sujeito frente a sua história, cultura e cuidado à saúde. Ao buscar-se uma perspectiva avaliativa da qualidade de vida, com a consideração de suas dimensões, isso significou a predominância no meio acadêmico da utilização de instrumentos com enfoque multidimensional e quantitativo.

No entanto, a associação com abordagens qualitativas parece ser bem-vinda não somente na análise do material advindo da aplicação dos instrumentos, mas na associação com outras técnicas complementares no momento de triangulação dos questionários padronizados. Daí aponta-se a possibilidade de, principalmente com o segmento de crianças e jovens, serem utilizadas 
técnicas complementares como grupos focais, observação participante, propostas lúdicas, a fim de melhor explorar dimensões interacionais, facilitando o acesso e respeito à experiência por eles desenvolvida.

Outro ponto que merece destaque diz respeito ao cuidado e rigor na utilização do termo qualidade de vida, em muito identificado a uma locução ampliada em significados globais sobre saúde, e por isso mesmo poucas vezes definida como merece. Essas definições são necessárias no processo de produzir conhecimento crítico e reflexivo, evitando seu uso apenas como atribuição de valor de marca, positivo. Esse tipo de apropriação muito comum com determinados conceitos advindos de campos mestiços - cultura, religião, sociedade, grupalidade, percepção, saúde, enfim vida - não permite o reconhecimento da imperiosa necessidade de definir-se e situarse os interesses e objetivos dos pesquisadores com suas pesquisas. O conceito de qualidade de vida evoca em sua origem e surgimento a necessidade das leituras e abordagens interdisciplinares no trato com as questões da saúde. Dessa forma, sua análise aplicada aos processos de saúde e doença de jovens e crianças precisa ser aprimorada principalmente na abordagem metodológica à custa de perder-se sua perspectiva ampliada, e fragmentarem-se sujeitos e experiências, reduzindo-os a relações entre variáveis.

Assim, faz-se necessária a criação e a utilização de instrumentos de avaliação de qualidade de vida para crianças e adolescentes que valorizem a perspectiva dos mesmos sobre sua experiência de adoecimento através de instrumentos adequados a sua fase de desenvolvimento.

\section{Colaboradores}

AHR Soares e AJ Martins foram responsáveis pela condução, análise da pesquisa, orientação metodológica e teórica, redação e revisão final do texto. MCB Lopes e JAA Brito foram responsáveis pela análise da pesquisa. CQ Oliveira foi responsável pela analise da pesquisa e redação do texto. MCN Moreira foi responsável pela redação e revisão do texto final. 


\section{Referências}

1. Bittar OJNV. Retorno ao trabalho após revascularização do miocárdio. Rev Saude Publica 1993; 27(3): 195-203.

2. Silva RBP, Ramalho AS, Cassorla RMS. A anemia falciforme como problema de saúde pública no Brasil. Rev Saúde Pública 1993; 27(1):54-58.

3. Soares AHR. Vocês riem porque eu sou diferente, eu rio porque vocês são todos iguais: as dimensões da qualidade de vida em jovens portadores de espinha bífida [tese]. Rio de Janeiro (RJ): Fundação Oswaldo Cruz; 2005.

4. Marconi MA, Lakatos EM. Metodologia do trabalho científico: procedimentos básicos, pesquisa bibliográfica, projeto e relatório, publicações e trabalhos científicos. $7^{\text {a }}$ ed. São Paulo: Atlas; 2008.

5. Dóro MP, Pasquini R, Löhr SS. A functional assessment of adolescents who were recipients of bone marrow transplantation: a prospective study. Rev Bras Hematol. Hemoter. 2003; 25(1):5-15.

6. Feitosa S, Colares V, Pinkham J. The psychosocial effects of severe caries in 4-year-old children in Recife, Pernambuco, Brazil. Cad Saude Publica 2005; 21(5):1550-1556.

7. Maia Filho HS, Costa CRM, Gomes MM. Epilepsia e saúde mental na infância. J. epilepsy clin. neurophysiol. 2006; 12(2):79-88.

8. Cartana MHF. Às vezes eles vão...: compreendendo qualidade de vida e promoção da saúde sob a ótica de uma mãe de pré-adolescentes. Rev LatinoAm Enfermagem 2005; 13(n. esp.):1177-1184.

9. Ludueña AC. Promoción de salud y calidad de vida en madres de preadolescentes. Una etnografia enfocada. Rev Latino-Am Enfermagem 2005; 13(n. esp.):1127-1134.

10. Soares AHR, Moreira MCN, Monteiro LMC, Pohl HG. A qualidade de vida de jovens portadores de espinha bífida do Children's National Medical Center - Washington DC. Cien Saude Colet 2006; 11(3):817826.

11. Minayo MCS. O desafio do conhecimento: pesquisa qualitativa em saúde. São Paulo: Hucitec/ Rio de Janeiro: Abrasco; 1999.

12. Silva VC, Leite AJM. Qualidade de vida em crianças com distúrbios obstrutivos do sono: avaliação pelo OSA-18. Rev Bras Otorrinolaringol. 2006; 72(6):747756.

13. Yacubian-Fernandes A, Ducati LG, Silva MV, Abramides DVM, Perosa GB, Palhares A, Gabarra RC, Giglio A, Portela L, Marinello JLP, Plese JPP, Zanin SA. Síndrome de crouzon: fatores envolvidos no desenvolvimento neuropsicologico e na qualidade de vida. Arq Neuro-Psiquiatr 2007; 65(2b):467-471.

14. Assumpção Jr. FB, Kuczynski E, Sprovieri MH, Aranha EMG. Escala de avaliação de qualidade de vida (AUQEI - Autoquestionnaire Qualité de Vie Enfant Imagé): validade e confiabilidade de uma escala para qualidade de vida em crianças de 4 a 12 anos. Arq Neuro-Psiquiatr 2000; 58(1):119-127.

15. Elias AV, Assumpção Jr FB. Qualidade de vida e autismo. Arq Neuro-Psiquiatr 2006; 64(2a):295-299.

16. Di Francesco RC, Fortes FSG, Komatsu CL. Melhora da qualidade de vida em crianças após adenoamigdalectomia. Rev Bras Otorrinolaringol. 2004; 70(6):748-751.
17. Faleiros FTV, Machado NC. Avaliação da qualidade de vida relacionada à saúde em crianças com distúrbios funcionais da defecação. J. Pediatr. 2006; 82(6):421-425.

18. Rozov T, Cunha MT, Nascimento O, Quittner AL, Jardim JR. Validação lingüística dos questionários de qualidade de vida em fibrose cística. J. Pediatr 2006; 82(2):151-156.

19. La Scala CSK, Naspitz CK, Sole D. Adaptação e validação do Pediatric Asthma Quality of Life Questionnaire (PAQLQ-A) em crianças e adolescentes brasileiros com asma. J Pediatr 2005; 81(1):54-60.

20. Barreire SG, Oliveira OA, Kazama W, Kimura M, Santos VLCG. Qualidade de vida de crianças ostomizadas na ótica das crianças e das mães. J. Pediatr. 2003; 79(1):55-62.

21. Maia Filho HS, Gomes MMF, Costa LM. Development and validation of a health related quality of life questionnaire for Brazilian children with epilepsy: preliminary findings Ar Neuro-Psiquiatr 2005; (63):389-394

22. Guimarães CA, Souza EAP, Montenegro MA, Cendes F, Guerreiro MM. Cirurgia para epilepsia na infância: avaliação neuropsicológica e de qualidade de vida. Arq Neuro-Psiquiatr 2003; 61(3B):786-792.

23. Fernandes PT, Souza EAP. Inventário simplificado de qualidade de vida na epilepsia infantil: primeiros resultados. Arq Neuro-Psiquiatr 1999; 57(1):40-43.

24. Souza EAP. Qualidade de vida na epilepsia infantil. Arq Neuro-Psiquiatr 1999; 57(1):34-39.

25. Costa MCO, Bigras M. Mecanismos pessoais e coletivos de proteção e promoção da qualidade de vida para a infância e adolescência. Cien Saude Colet 2007; 12(5):1101-1109.

26. Martins LM, França APD, Kimura M. Qualidade de vida de pessoas com doença crônica. Rev LatinoAm Enfermagem 1996; 4(3):5-18.

27. Brasil TB, Ferriani VPL, Machado, CSM. Inquérito sobre a qualidade de vida relacionada à saúde em crianças e adolescentes portadores de artrites idiopáticas juvenis. J. Pediatr. 2003; 79(1):63-68.

28. Favarato MECS, Romano BW. Cirurgia cardíaca na infância: repercussöes na qualidade de vida do adolescente/Heart surgery in childhood: impact on the quality of life of adolescents Arq bras Cardial 1994; 62(3):171-174.

29. Bampi LNS, Guilhem D, Lima DD. Qualidade de vida em pessoas com lesão medular traumática: um estudo com o WHOQOL-bref. Rev bras epidemiol 2008; 11(1):67-77.

30. Novato TS, Grossi SAA, Kimura M. Adaptação cultural e validação da medida "Diabetes Quality of Life for Youths" de Ingersoll e Marrero para a cultura brasileira. Latino-Am Enfermagem 2008; 16(2): 224-230.

31. Anders JC, Lima RAG. Crescer como transplantado de medula óssea: repercussóes na qualidade de vida de crianças e adolescentes. Rev Latino-Am Enfermagem 2004; $12(6): 866-874$.

32. Radünz V, Olson J. Promoção de saúde e qualidade de vida entre mães de pré-adolescentes: um estudo etnográfico enfocado em Timbó/SC-Brasil. Rev Latino-Am Enfermagem 2005; 13(n. esp.):1135-1141. 
33. Soares AHR, Moreira MCN, Monteiro LMC. Jovens portadores de deficiência: sexualidade e estigma Cien Saude Colet 2008; 13(1):185-194.

34. Seidl EMF, Zannon CMLC. Qualidade de vida e saúde: aspectos conceituais e metodológicos. Cad. Saude Publica 2004; 20(2):580-588.

35. Costa R, Silva KR, Mendonça RC, Nishioka S, Siqueira S, Tamaki WT, Crevelari ES, Moreira LFP, Martinelli Filho M. Incidência de choques e qualidade de vida em jovens com cardioversor-desfibrilador implantável. Arq Bras Cardiol. 2007; 88(3):258-264.

36. Monteiro CA, D'aquino BMH, Iunes R, Gouveia NC, Taddei JAAC, Cardoso MAA. ENDEF e PNSN: para onde caminha o crescimento físico da criança brasileira? Cad. Saude Publica 1993; 9(Supl. 1):S85-S95.

37. Chaves CRMM, Oliveira CQ, Britto JAA, Elsas MICG. Exercício aeróbico, treinamento de força muscular e testes de aptidão física para adolescentes com fibrose cística: revisão da literatura. Rev Bras Saude Mater Infant 2007; 7(3):245-250.

38. Oria H. Creches: perspectivas atuais/Nurseries: actual perspectives Rev paul pediatr 1988; 6(23):141-8.

39. Oliveira CJ, Pereira CAR, Pontes JNCP, Fialho AVM, Moreira TMM. Análise da produção cientifica na área da saúde sobre qualidade de vida no Brasil entre 2000 e 2005: um estudo bibliográfico. Revista Eletrônica de Enfermagem 2007; 9(2):496-505.

40. The WHOQOL Group. The World Health Organization Quality of Life Assessment (WHOQOL): position paper from the World Health Organization. Social Science and Medicine 1995; 41(10):1403-1409.

41. Fleck MP, Louzada S, Xavier M, Cachamovich E, Vieira G, Santos L, Pinzon V. Aplicação da versão em português do instrumento abreviado de avaliação da qualidade de vida WHOQOL-bref. Rev Saude Publica 2000; 34(2):350-356.

42. Auquier P, Simeoni MC, Mendizabal H. Approches théoriques et méthodologiques de la qualité de vie liée à la santé. Revue Prevenir 1997; 33(1):77-86.

43. Minayo MCS, Hartz ZMA, Buss PM. Qualidade de vida e saúde: um debate necessário. Cien Saude Colet 2000; 5(1):7-18.
44. Fleck MPA, Leal OF, Louzada S, Xavier M, Chachamovich E, Vieira G, Santos L, Pinzon V. Desenvolvimento da versão em português do instrumento de avaliação de qualidade de vida da OMS (WHOQOL-100). Rev Bras Psiquiatr 1999; 21(1):19-28.

45. Salgado PCB, Souza EA. Qualidade de vida em epilepsia e percepção de controle de crises. Arq Neuro-Psiquiatr 2001; 59(3A):537-540.

46. Salgado PCB, Souza EA. Variáveis psicológicas envolvidas na qualidade de vida de portadores de epilepsia. Estud. psicol. (Natal), 2003; 8(1):165-168.

47. Silva JH, Olson J. Promoción de salud y calidad de vida en madres de preadolescentes de la comunidad de Chiguayante-Chile: Una etnografia enfocada. Rev Latino-Am Enfermagem 2005; 13(n. esp.): 1164-1168.

48. Rogers W, Ballantyne A. Populações especiais: vulnerabilidade e proteção. Texto do Curso de Atualização à Distância em Ética em Pesquisa. In: Anais - Instituto de Bioética, Direitos Humanos e Gênero; 2008; Brasília.

49. Mello DB, Moreira MCN. A hospitalização e o adoecimento pela perspectiva de crianças e jovens portadores de fibrose cística e osteogênese imperfeita. Cien Saude Colet 2010; 15(2):453-461.

Artigo apresentado em 28/11/2008

Aprovado em 27/04/2009

Versão final apresentada em 15/04/2009 